\title{
Numerical Simulation of Quantum Logic Gates Based on Quantum Wires
}

\author{
A. BERTONI ${ }^{\mathrm{a}, *}, \mathrm{P} . \mathrm{BORDONE}^{\mathrm{a}}, \mathrm{R} \cdot \mathrm{BRUNETTI}^{\mathrm{a}}$, \\ C. JACOBONI ${ }^{\mathrm{a}}$ and S. REGGIANI ${ }^{\mathrm{b}}$ \\ ${ }^{a}$ Istituto Nazionale per la Fisica della Materia, Dipartimento di Fisica, \\ Università di Modena e Reggio Emilia, Via Campi 213/A, 41100 Modena, Italy; \\ ${ }^{\mathrm{b}}$ DEIS, Università di Bologna, Viale Risorgimento 2, Bologna, Italy
}

\begin{abstract}
A system based on frontier mesoscopic semiconductor technology, able to perform the basic quantum operations needed for quantum computation, is proposed. The elementary quantum bit (qubit) is defined as the state of an electron running along a couple of quantum wires coupled through a potential barrier with variable height and/ or width. A proper design of the system, together with the action of Coulomb interaction of two electrons representing two different qubits, allows the implementation of basic one-qubit and two-qubit quantum logic gates. Numerical simulations confirm the correctness of the hypothesis.
\end{abstract}

Keywords: Quantum computing; Quantum wires; Single electrons devices; Coherent transport

\section{INTRODUCTION}

Quantum theory of computation has been rapidly developed in the recent years. Still it seems very difficult to find a quantum system suitable for realization of the fundamental logic gates, technologically feasible and easily integrable into traditional circuitry. Furthermore, since many qubits are necessary to manipulate quantum information, the physical system which represents the qubit must be reliable and easily reproducible with high quality standards even for structures with a large number of units. The main difficulty in many cases is the onset of interactions between the system and the environment which produce decoherence and consequent loss of information.

As in classical information theory, two-state $(|0\rangle$ and $|1\rangle)$ systems are required in order to encode and process information. In quantum computation, however, the bit can be in any superposition of the two states. Furthermore, if a pair of twostate particles is considered, states can be formed for the whole system with linear combinations of products of single-particle states. In these two-particle states the single-particle states are "entangled" and, as a consequence, none of the

*Corresponding author. Tel.: + 39059 2055292, e-mail: bertoni.andrea@unimo.it 
individual systems carries any information in itself; all the information is instead contained in the system of the two qubits.

\section{ONE-QUBIT GATES}

In our proposal two identical semiconductor quantum wires separated by a potential barrier constitute the device for the two-state quantum system (see Fig. 1). The potential profile along the $x$ direction is a double well with the two lower eigenfunctions $\left(\psi_{\mathrm{e}}\right.$ and $\left.\psi_{0}\right)$ having even and odd symmetry. It is well known that an electron localized in one of two coupled quantum wells oscillates between them with a period $\tau=\mathrm{h} /\left(\varepsilon_{0}-\varepsilon_{\mathrm{e}}\right)$, where $\varepsilon_{\mathrm{e}}$ and $\varepsilon_{0}$ are $\psi_{\mathrm{e}}$ and $\psi_{0}$ energies, respectively. In our case, the barrier height and/or width between the wells changes along the $y$ direction. While the electron coherently propagates along the wires it experiences a different coupling between them. This allows the realization of an assigned transfer process by means of a suitable heterostructure design [1].

The qubit states $|0\rangle$ and $|1\rangle$ are defined as the localization of the electron in the left and right wire respectively. They represent the linear combinations of the even and odd electron states corresponding to the lowest energy levels of the quantized (transversal) direction:

$$
|0\rangle=\frac{1}{\sqrt{2}}\left(\left|\psi_{e}\right\rangle+\mid \psi_{o}\right), \quad|1\rangle=\frac{1}{\sqrt{2}}\left(\left|\psi_{e}\right\rangle-\left|\psi_{o}\right\rangle\right)
$$

It is known in the literature [2] that a set of gates consisting of all one-qubit rotations (rotations of $\mathrm{U}(2)$ group) and any non trivial two-qubit gate, is universal in the sense that all unitary operations on $\mathrm{n}$ qubits can be expressed as a composition of these gates.
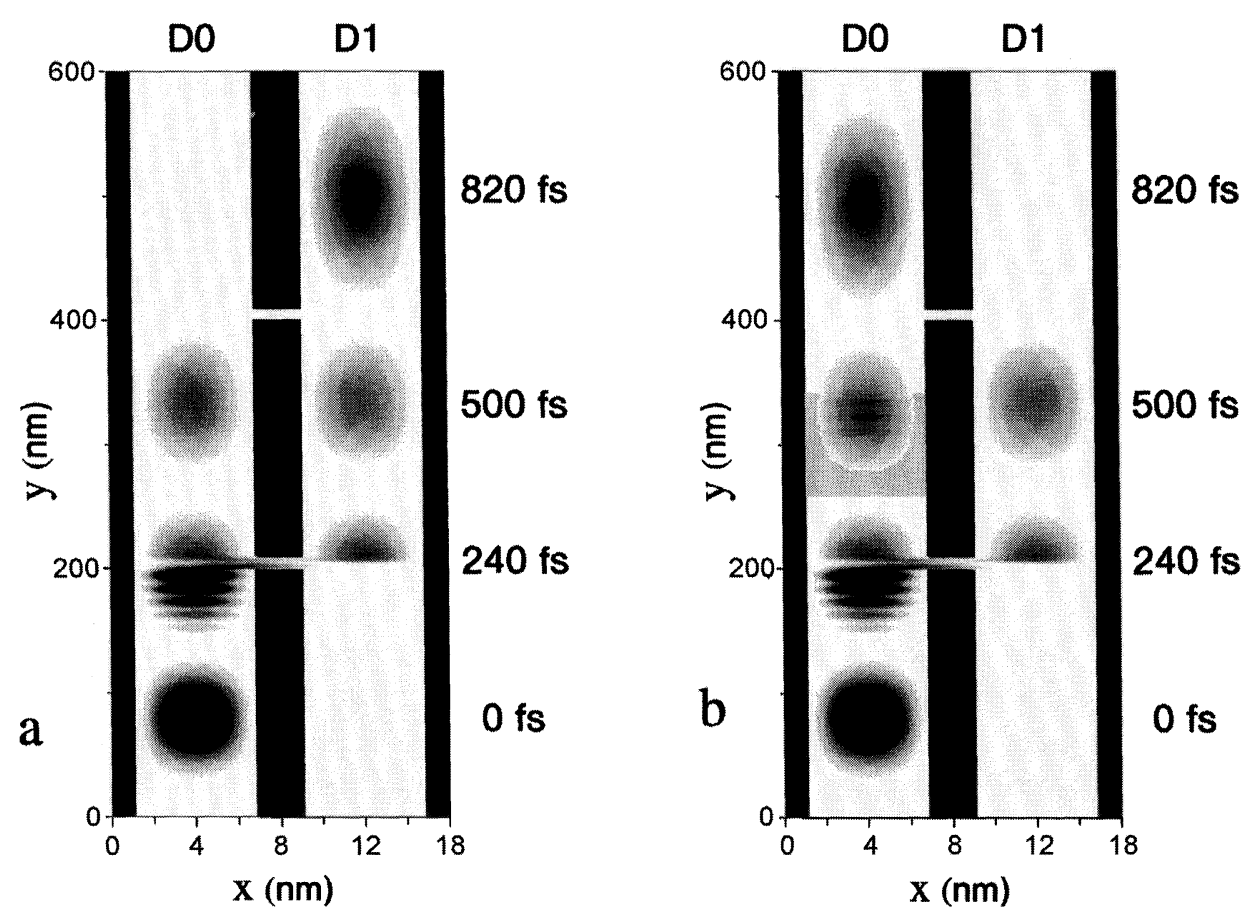

FIGURE 1 Electron density at different times in a coupled quantum wires device: (a) two electronic beam splitters realize a quantum NOT operation, (b) two electronic beam splitters with a delaying potential between them in one of the wires (grey region in D0) realize an identity gate. The delay is realized with a potential barrier $80 \mathrm{~nm}$ long and $10 \mathrm{meV}$ high. The electron is injected from the bottom of the figure with a kinetic energy of $0.1 \mathrm{eV}$. 
A general one-qubit rotation can be decomposed in four trasformations:

$$
\mathbf{U}(\delta, \alpha, \beta, \theta)=e^{i \delta} \mathbf{R}\left(\alpha-\frac{\pi}{2}\right) \mathbf{S}(\theta) \mathbf{R}\left(\beta+\frac{\pi}{2}\right)
$$

where $\delta$ is a phase-shift factor (any global phase factor will be, in the following, neglected), $\mathbf{R}(\phi)$ is expressed as

$$
\mathbf{R}(\phi)=\left(\begin{array}{cc}
e^{i(\phi / 2)} & 0 \\
0 & e^{-i(\phi / 2)}
\end{array}\right)
$$

and $\mathbf{S}(\theta)$ is given by

$$
\mathbf{S}(\theta)=\left(\begin{array}{rr}
\cos (\theta / 2) & i \sin (\theta / 2) \\
i \sin (\theta / 2) & \cos (\theta / 2)
\end{array}\right)
$$

The above transformations have been realized in the frame of the proposed system. The $\mathbf{R}(\phi)$ transformation is obtained by introducing a suitable potential barrier in one of the wires that slightly delays the propagation of the wave packet along that wire. Such a delay introduces a phase difference in the two parts $(|0\rangle$ and $|1\rangle)$ of the wave function of the propagating electron. The $\mathbf{S}(\theta)$ transformation represents the evolution of the electron wave function crossing a coupling window, e.g., a region with a lower potential barrier between the wires. A desired value of $\theta$ is obtained with a suitable choice of the window length along the wires.

As a first example of one-qubit gate, in Figure 1a a quantum NOT gate is shown, realized with the successive application of two beam splitter corresponding to two $\mathbf{S}(\pi / 2)$ transformations. In Figure $1 \mathrm{~b}$ the identity operation is obtained by applying the transformation $\mathbf{S}(\pi / 2) \mathbf{R}(\pi) \mathbf{S}(\pi / 2)$. The device depicted in Figure 1 behaves as a controlled-NOT (CNOT) gate: the logic state of the qubit is controlled by the presence of the potential applied to the left wire. It is worth noting that this gate is not a fully quantum CNOT, since the delaying potential could not represent a qubit.

\section{TWO-QUBITS GATES}

To complete the set of universal quantum gates, a non trivial two-qubits gate is needed [3]. A quantum CNOT gate is proposed in which the Coulomb potential generated by an electron (control qubit) allovs to control the state of a second electron (data qubit). A NOT gate is first realized by means of a $\mathbf{S}(5 \pi)$ transformation. This is obtained with a coupling window between the wires corresponding to five half periods of oscillation of the electron wave function. The functionality of the NOT gate is preserved also for this case, as confirmed by numerical, simulations. Another couple of wires (the control qubit) is added in a plane above the plane of the NOT gate. One of them ( $\mathrm{CO})$ is located exactly above the barrier separating the two wires of the NOT gate at a distance such that Coulomb interaction between the two electrons is effective. The second wire of the control qubit (C1) is located far enough from the other three in order to prevent any Coulomb interaction or electron transfer.

If the control qubit is set to 1 (the above electron propagates in wire $\mathrm{C} 1$ ) the Coulomb interaction between the two particles is very weak and does not affect the NOT operation performed by the $\mathbf{S}$ gate on the electron representing the data qubit (Fig. 2a). If the control qubit is set to 0 (the above electron propagates in wire $\mathrm{C} 0$ ) it is possible, with an appropriate choice of the electrons injection timings, to obtain a slowing down of the data electron due to Coulomb interaction. In the simulations described in the next section, the physical parameters of the system and the anticipation of the control electron have been properly designed so that the data electron crosses the coupling window region in a time equal to six half periods of oscillation, realizing a $\mathbf{S}(6 \pi)$ gate, i.e., the identity (Fig. 2b). It is straightforward to verify that the structure described above realizes 

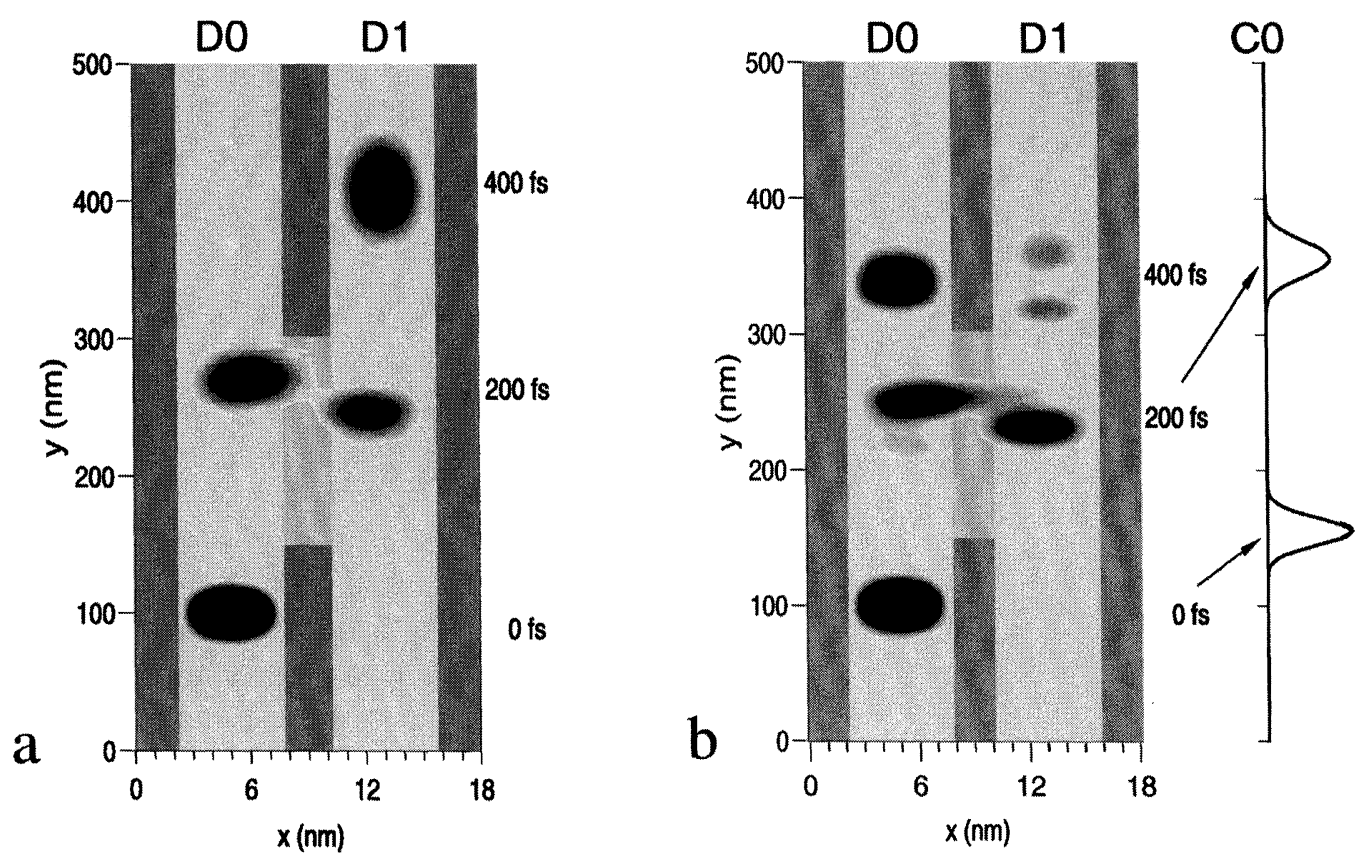

FIGURE 2 Electron density in the two wires of the data qubit at different times for the CNOT gate. The control qubit, not shown in the picture, consists of two not-coupled quantum wires parallel to the data-qubit wires and lying in a xy plane $4 \mathrm{~nm}$ distant from the plane of the figure (see text). In case (a) the logic state of the control qubit is $|1\rangle$ so the data qubit changes from $|0\rangle$ to $|1\rangle$ in case (b) the control qubit (whose evolution is shown in the one dimensional graph on the side) is in state $|0\rangle$ and prevents the data qubit to change his state.

the two-qubit quantum CNOT operation. A more detailed description of the device is presented in Ref. [4].

A second two-qubit gate proposed is based on the effect shown in Figure 1b. The delay in the propagation along the wires of the electron wave packet, is obtained by means of the presence of a second electron propagating along another couple of wires in the same plane (see Fig. 3). The system is designed in such a way that only if the two electrons propagates in adjacent wires ( $\mathrm{C} 1$ and D1), the mutual Coulomb interaction is able to produce an actual effect. What is obtained is a "conditional phase shifter" (CPS, one of the fundamental gates in quantum information theory) whose matrix representation is

$$
\mathbf{T}(\alpha)=\left(\begin{array}{cccc}
1 & 0 & 0 & 0 \\
0 & 1 & 0 & 0 \\
0 & 0 & 1 & 0 \\
0 & 0 & 0 & e^{i \alpha}
\end{array}\right)
$$

where the basis states are $|00\rangle,|01\rangle,|10\rangle,|11\rangle$. The rotation angle $\alpha$ can be selected with a proper lenght of the interaction region, i.e., the region in which the adjacent wires of the two different qubits are at low distance.

To show the gate functionality, a device implementing the transformation

$$
\left(\begin{array}{ccc}
\mathbf{S}(\pi / 2) & 0 & 0 \\
& 0 & 0 \\
0 & 0 & \\
0 & 0 & \mathbf{S}(\pi / 2)
\end{array}\right) \mathbf{T}(\pi)\left(\begin{array}{ccc}
\mathbf{S}(\pi / 2) & 0 & 0 \\
& 0 & 0 \\
0 & 0 & \\
0 & 0 & \mathbf{S}(\pi / 2)
\end{array}\right)
$$

is presented in Figure 3. Equation (5) represents the application of: (1) a beam splitter applied to the data qubit, (2) a CPS acting on the couple of qubits, (3) again a beam splitter on the data qubit. 

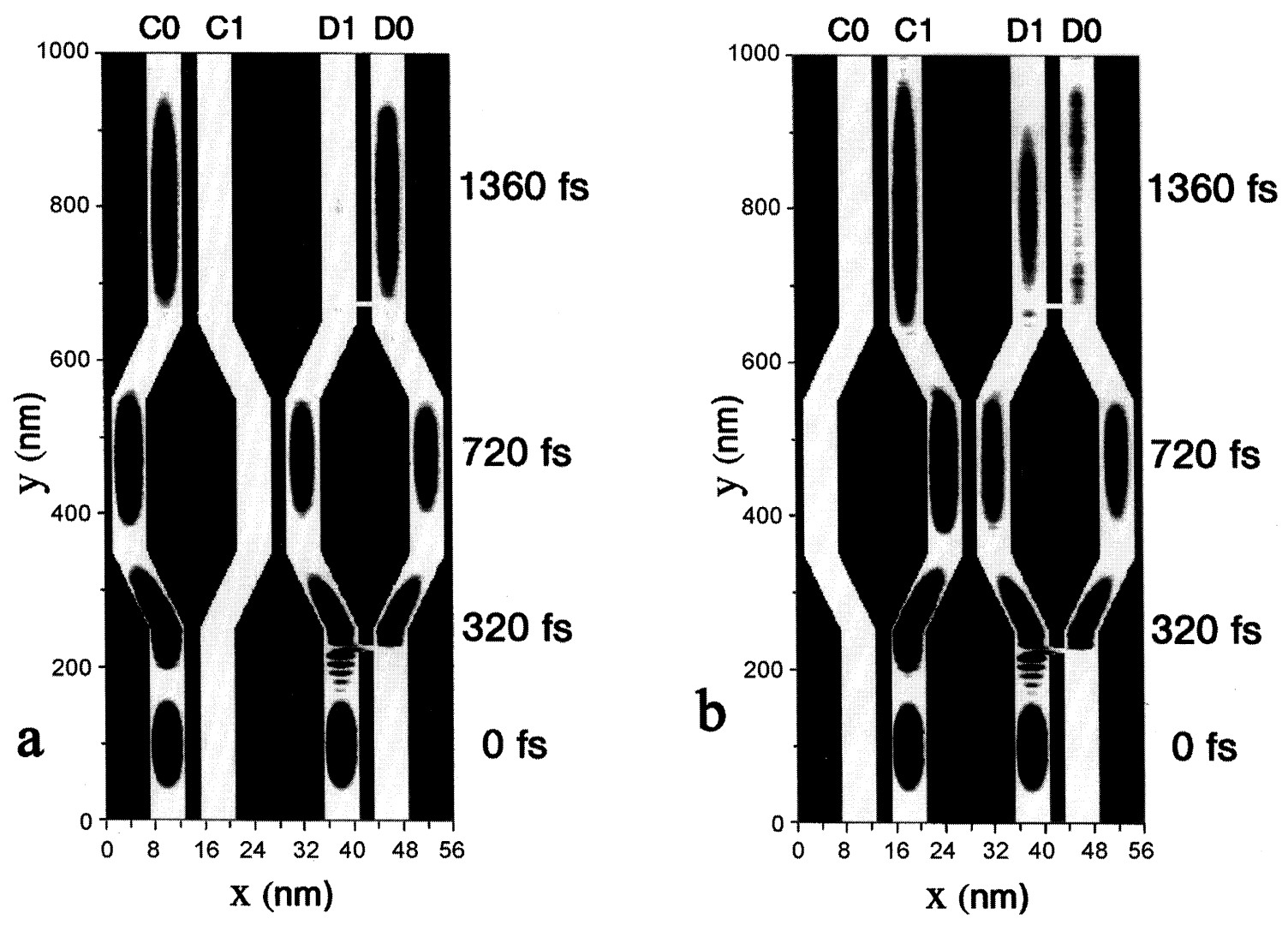

FIGURE 3 Electron density at different times in a quantum wires device implementing the two-qubit operation described by Eq. (6). In (a) the Coulomb interaction between the electrons is not effective due to the large distance, so the input state $|01\rangle$ is evolved in $|00\rangle$ by means of two beam splitters acting on the data-electron; in (b) the input state $|11\rangle$ is preserved by means of the following mechanism: (a) an electronic beam splitter (see text) equally splits the data-electron wave function, (b) the Coulomb interaction between electrons delays the wave functions propagating in $\mathrm{D} 1$ and $\mathrm{C} 1$ wires, (c) the two parts of data-electron wave function reach the second beam splitter with a phase shift and the electron propagates in D1 wire.

\section{NUMERICAL SIMULATIONS}

The systems proposed have been studied by solving numerically the $2 \mathrm{D}$ time dependent Schrödinger equation of the electrons. A bidimensional rectangular grid with $\Delta x=0.25 \mathrm{~nm}$ and $\Delta y=0.5 \mathrm{~nm}$ has been used for the discretization of both the structure potential and the electron wave functions. Simulations have been performed by means of a simple finite-difference relaxation method. It has been applied at each step of the time evolution performed in a CrankNicholson scheme with $\Delta t=0.2 \mathrm{fs}$. The electronelectron interaction is accounted for, at each time step, by means of an explicit calculation of the Coulomb potential generated by each electron added to the structure potential. The material parameters of GaAs have been used.

The lateral extension of single wires is $6 \mathrm{~nm}$. The confining potential (darker in figures) is assumed to be infinte. The initial condition for electron wave functions has been taken along the $x$ axis as the ground state of an infinite square well having the same lateral extension of the single wire, along the "free" $y$ direction as a minimum uncertainty wave packet with a kinetic energy of $0.1 \mathrm{eV}$. The total energy of the two electrons must be carefully tuned for the correct operation of the coupling 
window and must not exceed the energy of the first excited transversal state in the wire. This is so in order to ensure occupation only of the ground state (and to guarantee equal kinetic energy for the two electrons along the direction of the wires).

The results are excellent in the case of one-qubit gates, while, for two-qubits gates, the broadening of the wave functions due to Coulomb interaction produces residues that are small in the case of CNOT gate (see Fig. 2b) but that can jeopardize the gate functionality in the case of $\mathbf{T}$ gate of Eq. (4) (see Fig. 3b). The search for optimal parameters for the geometry of the system and for the appropriate initial energy of injected electrons is being performed.

The physical systems proposed in this paper should be already experimentally realizable and tested using frontier mesoscopic semiconductor technology. As last relevant consideration, the features of coherent electron propagation discussed above offer interesting and innovative possibilities also for single-electron devices applied to classical computation.

\section{Acknowledgments}

This work has been partially supported by MURST under the $40 \%$ Project on Quantum Computation and by the U.S. Office of Naval Research (contract n. N00014-98-1-0777).

\section{References}

[1] Kroemer, H. and Okamoto, H. (1984). Some design considerations for multi-quantum-wells lasers, Jap. J. of Appl. Phys., 23, 970-974.

[2] Barenco, A. et al. (1995). Elementary gates for quantum computation, Phys. Rev. A, 52, 3457-3467.

[3] Lloyd, S. (1995). Almost any quantum logic gate is universal, Phys. Rev. Lett., 75, 346-349.

[4] Bertoni, A. et al., Quantum logic gates based on coherent electron transport in quantum wires, Phys. Rev. Lett., in press. 

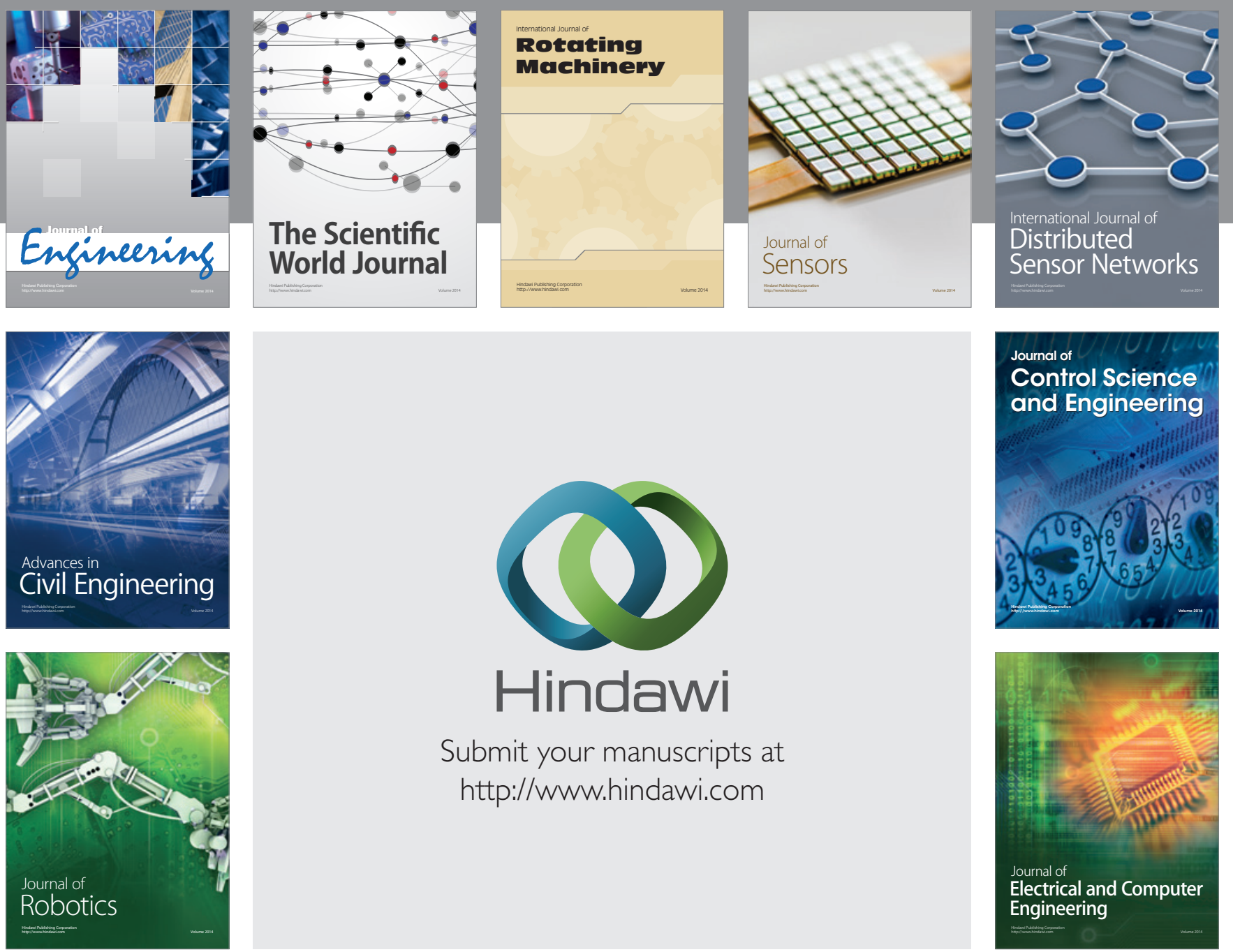

Submit your manuscripts at

http://www.hindawi.com
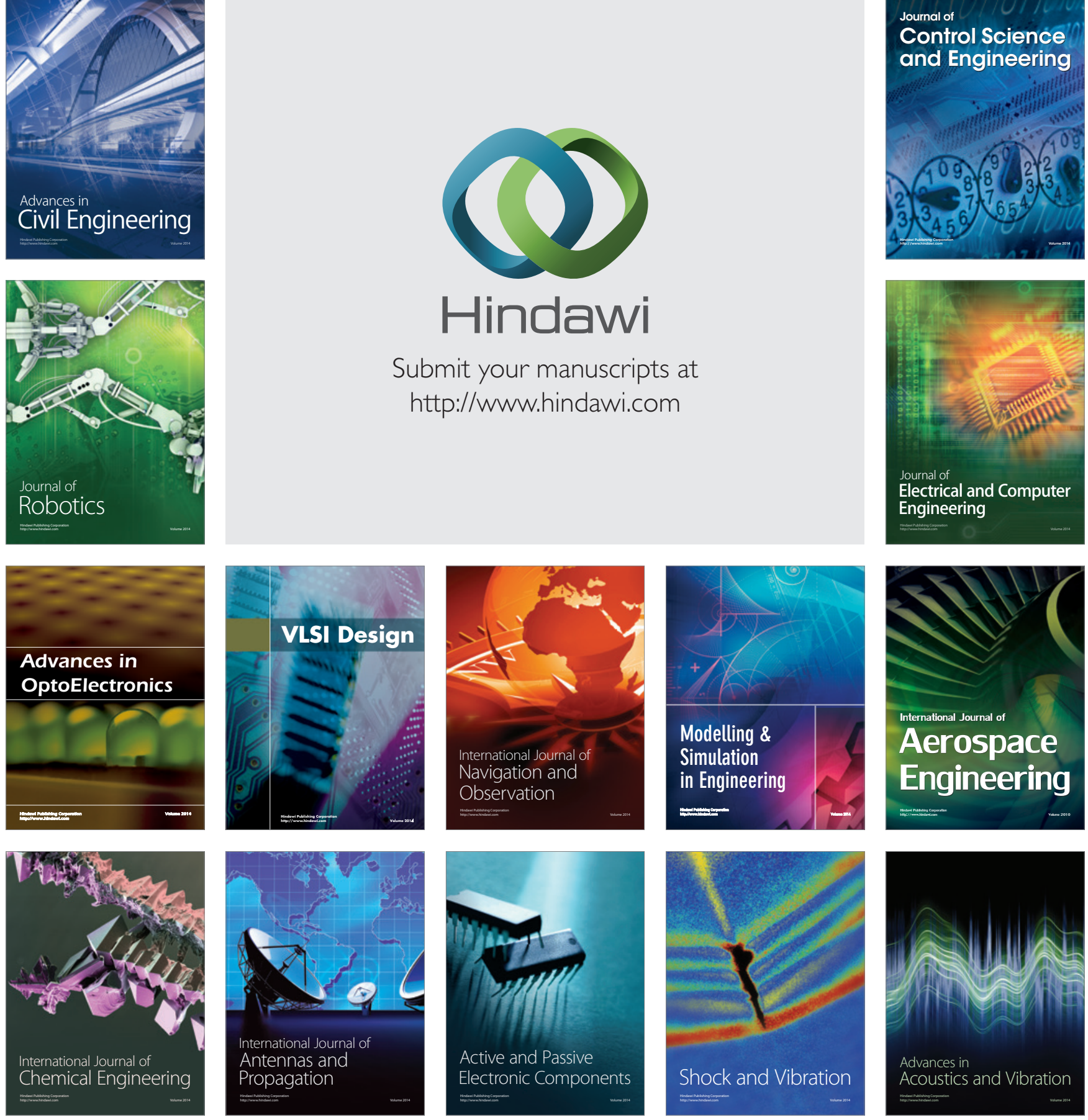